\title{
Evolution of Voltage-Dependent Anion Channel Function: From Molecular Sieve to Governator to Actuator of Ferroptosis
}

\author{
John J. Lemasters ${ }^{1,2 *}$ \\ ${ }^{1}$ Center for Cell Death, Injury and Regeneration, Department of Drug Discovery and Biomedical Sciences, Medical University \\ of South Carolina, Charleston, SC, United States, ${ }^{2}$ Department of Biochemistry and Molecular Biology, Medical University of \\ South Carolina, Charleston, SC, United States
}

OPEN ACCESS

Edited by:

Varda Shoshan-Barmatz,

Ben-Gurion University

of the Negev, Israel

Reviewed by:

M. K. Mathew,

National Centre for Biological

Sciences, India

Vito De Pinto,

Università degli Studi

di Catania, Italy

${ }^{*}$ Correspondence:

John J. Lemasters

jjlemasters@musc.edu

Specialty section:

This article was submitted to Molecular and Cellular Oncology,

a section of the journal

Frontiers in Oncology

Received: 01 October 2017 Accepted: 24 November 2017

Published: 19 December 2017

Citation:

Lemasters JJ (2017) Evolution of Voltage-Dependent Anion Channel

Function: From Molecular Sieve to Governator to Actuator of Ferroptosis.

Front. Oncol. 7:303. doi: 10.3389/fonc.2017.00303
The voltage-dependent anion channel (VDAC) is well known as the pathway for passive diffusion of anionic hydrophilic mitochondrial metabolites across the outer membrane, but a more complex functionality of the three isoforms of VDAC has emerged, as addressed in the Frontiers in Oncology Research Topic on "Uncovering the Function of the Mitochondrial Protein VDAC in Health and Disease: from Structure-Function to Novel Therapeutic Strategies." VDAC as the single most abundant protein in mitochondrial outer membranes is typically involved in isoform-specific interactions of the mitochondrion with its surroundings as, for example, during mitochondria-dependent pathways of cell death. VDAC closure can also act as an adjustable limiter (governator) of global mitochondrial metabolism, as during hepatic ethanol metabolism to promote selective oxidation of membrane-permeant acetaldehyde. In cancer cells, high free tubulin inhibits VDAC1 and VDAC2, contributing to suppression of mitochondrial function in the Warburg phenomenon. Erastin, the canonical inducer of ferroptosis, opens VDAC in the presence of tubulin and hyperpolarizes mitochondria, leading to mitochondrial production of reactive oxygen species, mitochondrial dysfunction, and cell death. Our understanding of VDAC function continues to evolve.

Keywords: erastin, ethanol, ferroptosis, reactive oxygen species, tubulin, voltage-dependent anion channel, Warburg phenomenon

In 1976, voltage-dependent anion channels (VDACs) were identified in mitochondria from Paramecium tetraurelia (1). Subsequent work, especially by Marco Colombini and his students, revealed VDAC to be a highly conserved $\sim 30 \mathrm{kDa}$ integral outer mitochondrial membrane protein that forms aqueous channels of $\sim 3 \mathrm{~nm}$ diameter in the open state, allowing passage of molecules up to $5 \mathrm{kDa}$ in size, although electrostatic profile is also an important determinant of channel permeance by charged molecules $(2,3)$. Consequently, VDAC is the common pathway for passive diffusion of all mostly anionic hydrophilic mitochondrial metabolites across the outer membrane. Thus, VDAC confers properties of a molecular sieve to the mitochondrial outer membrane, a long accepted and necessary attribute, but one that is, let's face it, a little boring.

Positive and negative membrane potentials symmetrically close VDAC to most metabolites with half maximal closure at $\pm 50 \mathrm{mV}$, indicating more complexity to VDAC functionality. With closure, pore diameter decreases to $1.8 \mathrm{~nm}$, which blocks movement of respiratory substrates, adenine nucleotides, and other molecules that move in and out of mitochondria during metabolism (4). In its 
closed state, VDAC becomes a relatively cation selective channel that nonetheless conducts small anions like $\mathrm{Cl}^{-}$(5). The question remains controversial whether voltage gating of VDAC is physiologically important, since Donnan potentials are too small for closure at the ionic strength of the intracellular milieu and because VDAC's own permeability to small electrolytes collapses metabolism-driven potentials. However, metabolite channeling within complexes of hexokinase, creatine kinase, the adenine nucleotide translocator (ANT), and VDAC has been suggested to form a positive inside outer membrane potential sufficient to close $\operatorname{VDAC}(6,7)$. Voltage gating may also be a fail-safe mechanism against loss of cellular metabolites should VDAC be inserted into the plasma membrane, as sometimes and somewhat controversially reported $(8,9)$, since cells have mechanisms to rapidly restore homeostasis of $\mathrm{Na}, \mathrm{K}, \mathrm{Ca}$, and $\mathrm{Cl}$ ions passing through "closed" VDAC, whereas homeostatic restoration of anionic metabolites released through "open" VDAC occurs much more slowly.

Each of the three VDAC isoforms (VDAC1, VDAC2, and VDAC3) has very similar structure, conductance, and voltagegating properties, but the existence of these different isoforms in humans and other mammalian species and their differential expression in various tissues suggests more functional complexity to VDAC (10-15). Since VDAC is the most abundant protein in the outer membrane, interactions of mitochondria with cytosolic proteins and other organelles typically involve VDAC, and generally speaking, these interactions are isoform specific. As well illustrated in this Frontiers in Oncology Research Topic on "Uncovering the Function of the Mitochondrial Protein VDAC in Health and Disease: from Structure-Function to Novel Therapeutic Strategies," specific VDAC isoforms are important for pathways signaling both apoptotic and non-apoptotic cell death $(16,17)$. Moreover, Reina and coworkers review new evidence of a unique role for VDAC3 cysteine over-oxidation as a mitochondrial oxidative marker participating in reactive oxygen species (ROS) signaling (18).

Scientists studying VDAC have long wondered whether and how changes of VDAC conductance might regulate mitochondrial function. Concrete evidence for this came from studies showing closure of VDAC at an early stage of apoptosis (19), but what about VDAC in cells that are not dying? It was then proposed that various degrees of VDAC closing can act as a dynamic limiter, or "governator," of global mitochondrial function (20). Such VDAC closure might account for unexplained anomalies of mitochondrial function seen in cells and tissues in certain contexts.

As an example, hepatic oxygen and ethanol metabolism nearly doubles within 2-3 h after gastric ethanol feeding, a so-called swift increase of alcohol metabolism (SIAM), which is an adaptive metabolic response to hasten the detoxification and elimination of both ethanol and its more toxic metabolite, acetaldehyde, by mostly alcohol dehydrogenase in the cytosol and aldehyde dehydrogenase in mitochondria $(21,22)$. Despite increased mitochondrial respiration, hepatic ATP paradoxically decreases by more than half after ethanol treatment but without activation of an identifiable ATPase. Moreover, steatosis occurs in parallel, indicative of inhibition of mitochondrial $\beta$-oxidation. To explain these phenomena, VDAC closure was proposed as a mechanism to inhibit mitochondrial release of ATP and uptake of fatty acylCoA, leading to cellular ATP depletion and steatosis, respectively. In addition, a protonophoric uncoupling pathway was hypothesized to open to stimulate respiration (20). Subsequent studies showed that the outer membranes of hepatocyte mitochondria do indeed become less permeable to adenine nucleotides and low molecular weight dextrans after ethanol and acetaldehyde treatment (23). Ethanol and acetaldehyde also decrease ureagenic respiration, a process requiring considerable flux of different metabolites through VDAC (24). Finally, intravital multiphoton microscopy reveals a reversible dose- and time-dependent depolarization of hepatocellular mitochondria after ethanol feeding in which depolarization occurs in an all-or-nothing fashion within any particular hepatocyte (25). Because small neutral aldehydes do not need VDAC or other carrier to cross the outer and inner membranes of mitochondria, VDAC closure and respiratory stimulation by uncoupling during SIAM together promote more rapid oxidation of membrane-permeant acetaldehyde while simultaneously inhibiting oxidation of competing substrates that require VDAC to enter mitochondria. These adaptations depend on hepatic aldehyde formation and revert as acetaldehyde is eliminated by oxidation to acetate. Similar mechanisms may also play a role in nonalcoholic steatohepatitis in which oxidative stress is an important component, since lipid peroxidation chain reactions generate aldehydes like malondialdehyde that close VDAC even more potently than acetaldehyde $(24,26)$.

Another example of a possible governator role for VDAC is the suppression of mitochondrial metabolism and enhancement of aerobic glycolysis that characterize the pro-proliferative Warburg metabolic phenotype of cancer cells. In this hypothesis, VDAC closure decreases mitochondrial ATP release, lowers cytosolic ATP/ADP, and thereby stimulates glycolysis (20). Hexokinase may be one molecule inhibiting VDAC, since hexokinase is overexpressed in cancer cells and inhibits VDAC reconstituted into planar lipid bilayers (27).

A more important VDAC closer in cancer cells is free dimeric tubulin, as reviewed in the Research Topic by Maldonado (28). Proliferating cancer cells have much higher levels of free tubulin compared to post-mitotic cells, because tubulin is needed for spindle formation at metaphase. Rostovtseva's group showed that low nanomolar free tubulin closes VDAC reconstituted into planar bilayers (29). VDAC1 and VDAC2, the most abundant isoforms in most cells, are sensitive to tubulin, whereas the least abundant VDAC3 is insensitive (14). In intact cancer cells, increased free tubulin after microtubule depolymerization causes mitochondrial membrane potential $(\Delta \Psi$, an indicator of mitochondrial metabolism) to decrease, apparently due to the inhibition of respiratory substrate entry through VDAC (30). By contrast, microtubule stabilization with paclitaxel, which decreases free tubulin, causes $\Delta \Psi$ to increase. Remarkably, the VDAC-binding molecule erastin blocks the inhibitory effect of free tubulin on VDAC inserted into lipid bilayers and reverses mitochondrial depolarization induced by elevated free dimeric tubulin in intact cancer cells $(14,31)$.

Erastin is the canonical inducer of ferroptosis, a type of nonapoptotic, oxidative cell death that is so-named because the iron 
chelator, desferal (deferoxamine), prevents erastin-induced cell killing (32). Desferal is well known to protect against many oxidative stresses, including ischemia-reperfusion, drug-induced hepatotoxicity, and exposure to oxidant chemicals (33-37). How then can VDAC opening be linked to oxidative stress? The answer is that mitochondrial ROS generation increases with increasing mitochondrial $\Delta \Psi$ and with increased reduction of the respiratory chain as respiratory substrates enter following VDAC opening (38-42). Thus, erastin and several erastin-like small molecules identified by high content screening increase mitochondrial ROS in parallel with mitochondrial hyperpolarization, as highlighted in Maldonado's review $(28,43,44)^{1}$. At later time points, ROS-dependent mitochondrial dysfunction occurs, which likely represents onset of the mitochondrial permeability transition. Ultimately cell death occurs, which is prevented by antioxidants like $N$-acetylcysteine. Thus, VDAC

${ }^{1}$ deHart DN, Fang D, Heslop K, Li L, Lemasters JJ, Maldonado EN. Opening of voltage dependent anion channels promotes reactive oxygen species generation, mitochondrial dysfunction and cell death in cancer cells. (2017) Submitted for publication.

\section{REFERENCES}

1. Schein SJ, Colombini M, Finkelstein A. Reconstitution in planar lipid bilayers of a voltage-dependent anion-selective channel obtained from paramecium mitochondria. J Membr Biol (1976) 30(2):99-120. doi:10.1007/ BF01869662

2. Colombini M. VDAC structure, selectivity, and dynamics. Biochim Biophys Acta (2012) 1818(6):1457-65. doi:10.1016/j.bbamem.2011.12.026

3. Colombini M, Mannella CA. VDAC, the early days. Biochim Biophys Acta (2012) 1818(6):1438-43. doi:10.1016/j.bbamem.2011.11.014

4. Rostovtseva T, Colombini M. VDAC channels mediate and gate the flow of ATP: implications for the regulation of mitochondrial function. Biophys $J$ (1997) 72(5):1954-62. doi:10.1016/S0006-3495(97)78841-6

5. Tan W, Colombini M. VDAC closure increases calcium ion flux. Biochim Biophys Acta (2007) 1768(10):2510-5. doi:10.1016/j.bbamem.2007.06.002

6. Lemeshko VV. VDAC electronics: 2. A new, anaerobic mechanism of generation of the membrane potentials in mitochondria. Biochim Biophys Acta (2014) 1838(7):1801-8. doi:10.1016/j.bbamem.2014.02.007

7. Lemeshko VV. VDAC electronics:3. VDAC-Creatine kinase-dependent generation of the outer membrane potential in respiring mitochondria. Biochim Biophys Acta (2016) 1858(7 Pt A):1411-8. doi:10.1016/j.bbamem.2016. 04.005

8. De Pinto V, Messina A, Lane DJ, Lawen A. Voltage-dependent anion-selective channel (VDAC) in the plasma membrane. FEBS Lett (2010) 584(9):1793-9. doi:10.1016/j.febslet.2010.02.049

9. Sabirov RZ, Merzlyak PG. Plasmalemmal VDAC controversies and maxianion channel puzzle. Biochim Biophys Acta (2012) 1818(6):1570-80. doi:10.1016/j.bbamem.2011.09.024

10. De Pinto V, Guarino F, Guarnera A, Messina A, Reina S, Tomasello FM, et al. Characterization of human VDAC isoforms: a peculiar function for VDAC3? Biochim Biophys Acta (2010) 1797(6-7):1268-75. doi:10.1016/j. bbabio.2010.01.031

11. Hiller S, Abramson J, Mannella C, Wagner G, Zeth K. The 3D structures of VDAC represent a native conformation. Trends Biochem Sci (2010) 35(9):514-21. doi:10.1016/j.tibs.2010.03.005

12. Messina A, Reina S, Guarino F, De Pinto V. VDAC isoforms in mammals. Biochim Biophys Acta (2012) 1818(6):1466-76. doi:10.1016/j.bbamem. 2011.10.005

13. Raghavan A, Sheiko T, Graham BH, Craigen WJ. Voltage-dependant anion channels: novel insights into isoform function through genetic models. Biochim Biophys Acta (2012) 1818(6):1477-85. doi:10.1016/j.bbamem.2011.10.019 opening is what Maldonado calls an "anti-Warburg pro-oxidant switch" that leads to reversion of the pro-proliferative Warburg metabolic phenotype of aerobic glycolysis, increased $\Delta \Psi$, mitochondrial ROS generation, and oxidative stress-induced ferroptoic cell death.

Overall, our understanding of VDAC function continues to evolve. No longer just a sieve allowing passive movement of small molecules across the mitochondrial outer membrane, VDAC is an active agent regulating interactions between mitochondria and their surroundings, global mitochondrial metabolism, and the life or death fate of cells. Not so boring after all.

\section{AUTHOR CONTRIBUTIONS}

$\mathrm{JJL}$ is responsible for the content of this article.

\section{FUNDING}

The author is supported by grants R01 AA021191, R01 CA184456, R01 DE016572, R01 DK073336, P20 GM103542, and P30 CA138313 from the National Institutes of Health.

14. Maldonado EN, Sheldon KL, DeHart DN, Patnaik J, Manevich Y, Townsend DM, et al. Voltage-dependent anion channels modulate mitochondrial metabolism in cancer cells: regulation by free tubulin and erastin. J Biol Chem (2013) 288(17):11920-9. doi:10.1074/jbc.M112.433847

15. Mertins B, Psakis G, Essen LO. Voltage-dependent anion channels: the wizard of the mitochondrial outer membrane. Biol Chem (2014) 395(12):1435-42. doi:10.1515/hsz-2014-0203

16. Karachitos A, Grobys D, Kulczynska K, Sobusiak A, Kmita H. The association of VDAC with cell viability of PC12 model of Huntington's disease. Front Oncol (2016) 6:238. doi:10.3389/fonc.2016.00238

17. Shoshan-Barmatz V, Krelin Y, Shteinfer-Kuzmine A, Arif T. Voltagedependent anion channel 1 as an emerging drug target for novel anti-cancer therapeutics. Front Oncol (2017) 7:154. doi:10.3389/fonc.2017.00154

18. Reina S, Guarino F, Magri A, De Pinto V. VDAC3 as a potential marker of mitochondrial status is involved in cancer and pathology. Front Oncol (2016) 6:264. doi:10.3389/fonc.2016.00264

19. Vander Heiden MG, Chandel NS, Li XX, Schumacker PT, Colombini M, Thompson CB. Outer mitochondrial membrane permeability can regulate coupled respiration and cell survival. Proc Natl Acad Sci U S A (2000) 97(9): 4666-71. doi:10.1073/pnas.090082297

20. Lemasters JJ, Holmuhamedov E. Voltage-dependent anion channel (VDAC) as mitochondrial governator - thinking outside the box. Biochim Biophys Acta (2006) 1762(2):181-90. doi:10.1016/j.bbadis.2005.10.006

21. Yuki T, Thurman RG. Swift increase in alcohol metabolism: time course and involvement of glycolysis. Biochem J (1980) 186:119-26. doi:10.1042/ bj1860119

22. Bradford BU, Rusyn I. Swift increase in alcohol metabolism (SIAM): understanding the phenomenon of hypermetabolism in liver. Alcohol (2005) 35(1):13-7. doi:10.1016/j.alcohol.2004.12.001

23. Holmuhamedov E, Lemasters JJ. Ethanol exposure decreases mitochondrial outer membrane permeability in cultured rat hepatocytes. Arch Biochem Biophys (2009) 481(2):226-33. doi:10.1016/j.abb.2008.10.036

24. Holmuhamedov EL, Czerny C, Beeson CC, Lemasters JJ. Ethanol suppresses ureagenesis in rat hepatocytes: role of acetaldehyde. J Biol Chem (2012) 287(10):7692-700. doi:10.1074/jbc.M111.293399

25. Zhong Z, Ramshesh VK, Rehman H, Liu Q, Theruvath TP, Krishnasamy Y, et al. Acute ethanol causes hepatic mitochondrial depolarization in mice: role of ethanol metabolism. PLoS One (2014) 9(3):e91308. doi:10.1371/ journal.pone.0091308

26. Holmuhamedov EL, Lovelace G, Lemasters JJ. Aldehyde products of ethanol oxidation and oxidative stress suppress ureagenic but not basal 
respiration of cultured hepatocytes. Biophys J(2011) 100(3):460a. doi:10.1016/j. bpj.2010.12.2704

27. Azoulay-Zohar H, Israelson A, Abu-Hamad S, Shoshan-Barmatz V. In selfdefence: hexokinase promotes voltage-dependent anion channel closure and prevents mitochondria-mediated apoptotic cell death. Biochem J (2004) 377(Pt 2):347-55. doi:10.1042/bj20031465

28. Maldonado EN. VDAC-tubulin, an anti-warburg pro-oxidant switch. Front Oncol (2017) 7:4. doi:10.3389/fonc.2017.00004

29. Rostovtseva TK, Sheldon KL, Hassanzadeh E, Monge C, Saks V, Bezrukov SM, et al. Tubulin binding blocks mitochondrial voltage-dependent anion channel and regulates respiration. Proc Natl Acad Sci U S A (2008) 105(48):18746-51. doi:10.1073/pnas.0806303105

30. Maldonado EN, Patnaik J, Mullins MR, Lemasters JJ. Free tubulin modulates mitochondrial membrane potential in cancer cells. Cancer Res (2010) 70(24):10192-201. doi:10.1158/0008-5472.CAN-10-2429

31. Yagoda N, von Rechenberg M, Zaganjor E, Bauer AJ, Yang WS, Fridman DJ, et al. RAS-RAF-MEK-dependent oxidative cell death involving voltagedependent anion channels. Nature (2007) 447(7146):864-8. doi:10.1038/ nature05859

32. Dixon SJ, Lemberg KM, Lamprecht MR, Skouta R, Zaitsev EM, Gleason CE, et al. Ferroptosis: an iron-dependent form of nonapoptotic cell death. Cell (2012) 149(5):1060-72. doi:10.1016/j.cell.2012.03.042

33. Farber JL. Mechanisms of cell injury by activated oxygen species. Environ Health Perspect (1994) 102(Suppl 10):17-24. doi:10.1289/ehp.94102s1017

34. Nieminen AL, Byrne AM, Herman B, Lemasters JJ. Mitochondrial permeability transition in hepatocytes induced by t-BuOOH: $\mathrm{NAD}(\mathrm{P}) \mathrm{H}$ and reactive oxygen species. Am J Physiol (1997) 272(4 Pt 1):C1286-94.

35. Kim JS, Jin Y, Lemasters JJ. Reactive oxygen species, but not Ca2+ overloading, trigger $\mathrm{pH}$ - and mitochondrial permeability transition-dependent death of adult rat myocytes after ischemia-reperfusion. Am J Physiol Heart Circ Physiol (2006) 290(5):H2024-34. doi:10.1152/ajpheart.00683.2005

36. Uchiyama A, Kim JS, Kon K, Jaeschke H, Ikejima K, Watanabe S, et al. Translocation of iron from lysosomes into mitochondria is a key event during oxidative stress-induced hepatocellular injury. Hepatology (2008) 48(5):1644-54. doi:10.1002/hep.22498

37. Kim JS, Wang JH, Lemasters JJ. Mitochondrial permeability transition in rat hepatocytes after anoxia/reoxygenation: role of $\mathrm{Ca} 2+$-dependent mitochondrial formation of reactive oxygen species. Am J Physiol Gastrointest Liver Physiol (2012) 302(7):G723-31. doi:10.1152/ajpgi.00082.2011

38. Dawson TL, Gores GJ, Nieminen AL, Herman B, Lemasters JJ. Mitochondria as a source of reactive oxygen species during reductive stress in rat hepatocytes. Am J Physiol (1993) 264(4 Pt 1):C961-7.

39. Korshunov SS, Skulachev VP, Starkov AA. High protonic potential actuates a mechanism of production of reactive oxygen species in mitochondria. FEBS Lett (1997) 416(1):15-8. doi:10.1016/S0014-5793(97)01159-9

40. Lemasters JJ, Nieminen AL. Mitochondrial oxygen radical formation during reductive and oxidative stress to intact hepatocytes. Biosci Rep (1997) 17(3): 281-91. doi:10.1023/A:1027332611839

41. Starkov AA, Fiskum G. Regulation of brain mitochondrial $\mathrm{H} 2 \mathrm{O} 2$ production by membrane potential and $\mathrm{NAD}(\mathrm{P}) \mathrm{H}$ redox state. JNeurochem (2003) 86(5):1101-7. doi:10.1046/j.1471-4159.2003.01908.x

42. Figueira TR, Barros MH, Camargo AA, Castilho RF, Ferreira JC, Kowaltowski AJ, et al. Mitochondria as a source of reactive oxygen and nitrogen species: from molecular mechanisms to human health. Antioxid Redox Signal (2013) 18(16):2029-74. doi:10.1089/ars.2012.4729

43. deHart DN, Gooz M, Rostovtseva T, Sheldon KL, Lemasters JJ, Maldonado E. Antagonists of the inhibitory effect of free tubulin on VDAC induce oxidative stress and mitochondrial dysfunction. Biophys J (2014) 106(2):591a. doi:10.1016/ j.bpj.2013.11.3272

44. deHart DN, Gooz M, Lemasters JJ, Maldonado E. Small anti-Warburg molecules kill hepatocarcinoma cells by opening voltage dependent anion channels and promoting mitochondrial oxidative stress. Hepatology (2015) 62(1 Suppl):373A. doi:10.1002/hep.28211

Conflict of Interest Statement: The author declares that the research was conducted in the absence of any commercial or financial relationships that could be construed as a potential conflict of interest.

Copyright $\odot 2017$ Lemasters. This is an open-access article distributed under the terms of the Creative Commons Attribution License (CC BY). The use, distribution or reproduction in other forums is permitted, provided the original author(s) or licensor are credited and that the original publication in this journal is cited, in accordance with accepted academic practice. No use, distribution or reproduction is permitted which does not comply with these terms. 